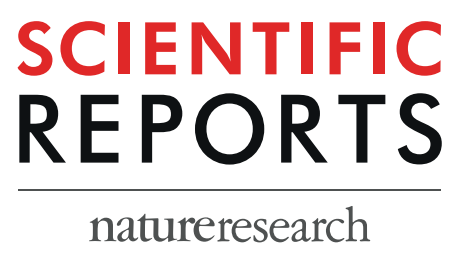

\title{
OPEN Cochlear Glucocorticoid Receptor and Serum Corticosterone Expression in a Rodent Model \\ of Noise-induced Hearing Loss: Comparison of Timing of Dexamethasone Administration
}

Received: 8 April 2019

Accepted: 14 August 2019

Published online: 02 September 2019

\author{
Seung-Hun Lee ${ }^{1}$, Ah-Ra Lyu ${ }^{1,2}$, Sun-Ae Shin ${ }^{1,2}$, Seong-Hun Jeong ${ }^{1,2}$, Sun-A Lee ${ }^{1,2}$, \\ Min Jung Park ${ }^{3}$ \& Yong-Ho Park ${ }^{1,3}$
}

Glucocorticoid (GC) is a steroid hormone secreted from the adrenal cortex in response to stress, which acts by binding to cytoplasmic glucocorticoid receptors (GRs). Dexamethasone (DEX) is a synthetic GC exhibiting immunosuppressive effects in both human and rodent models of hearing loss. While clinical evidence has shown the effectiveness of DEX for treatment of various inner ear diseases, its mechanisms of action and the optimal timing of treatment are not well understood. In the present study, intergroup comparisons were conducted based on the time point of treatment with DEX: (1) pretreatment; (2) posttreatment; and (3) pre\&post-noise. The pre\&post DEX treatment group showed a significant improvement in threshold shift at 1 day post-noise exposure as compared to the TTS (transient threshold shift)-only group at 8 and $16 \mathrm{kHz}$. Both TTS and PTS (permanent threshold shift) significantly reduced cochlear GR mRNA expression and increased serum corticosterone and cochlear inflammatory cytokines. The pre\&post DEX treatment group showed a significant decrease in serum corticosterone level as compared to other DEX treatment groups and TTS-treated group at 3 days after acoustic trauma. Our results suggest that the timing of DEX administration differentially modulates systemic steroid levels, GR expression and cochlear cytokine expression.

Glucocorticoids (GCs) are a class of steroid hormones secreted from the adrenal cortex in response to stress, which protect the organism against the negative effects of that stress ${ }^{1-4}$. They are among the most commonly prescribed drugs and are used for a wide range of medical conditions, including inner ear diseases, e.g., sudden idiopathic hearing loss ${ }^{5}$, acute noise-induced hearing $\operatorname{loss}^{6}$, and Ménière's disease ${ }^{7}$. GCs exert powerful antiinflammatory effects by inhibiting several inflammatory mediators and increasing cochlear blood flow, to prevent hair cell damage caused by inflammation and ischemia in the inner ear ${ }^{8-12}$.

The actions of GCs are predominantly mediated through glucocorticoid receptors (GRs). GRs are ubiquitously expressed throughout the body, including the inner ear. In rodents, GRs are highly expressed in the stria vascularis, inner hair cells, outer hair cells (OHCs), and spiral ligament of the cochlea and cochlear nerve ${ }^{13,14}$. In humans, the highest level of GR expression is found in the spiral ligament, with the lowest level seen in the saccule ${ }^{15}$. Hearing function has been reported to be closely related to GR expression ${ }^{11,15-19}$.

Dexamethasone (DEX), a synthetic GC, is widely used clinically due to its antiinflammatory, antishock, and immunosuppressive properties. As shown in our previous studies and confirmed by other groups, systemic or

${ }^{1}$ Department of Otolaryngology-Head and Neck Surgery, College of Medicine, Chungnam National University, Daejeon, Republic of Korea. ${ }^{2}$ Department of Medical Science, College of Medicine, Chungnam National University, Daejeon, Republic of Korea. ${ }^{3}$ Brain Research Institute, College of Medicine, Chungnam National University, Daejeon, Republic of Korea. Seung-Hun Lee and Ah-Ra Lyu contributed equally. Correspondence and requests for materials should be addressed to M.J.P. (email: mjpark@cnu.ac.kr) orY.-H.P. (email: parkyh@cnu.ac.kr) 


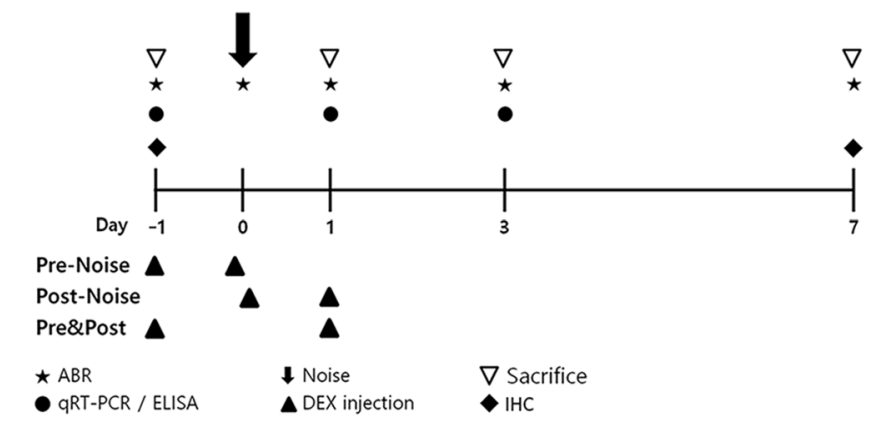

Figure 1. Schematic of the experimental design. Auditory brainstem response (ABR) thresholds were measured at four time points: prior to, immediately after, and 1, 3 and 7 days after noise exposure. Two dexamethasone (DEX) injections were given to animals in three treatment groups: (1) pre-noise group, injections at 1 day before and immediately prior to noise exposure; (2) post-noise group, injections immediately after and 1 day after noise exposure; and (3) pre\&post-noise group, injections at 1 day before and 1 day after noise exposure. Serum and/or tissue lysates were collected prior to and at 1,3, and 7 days after noise exposure for biochemical analyses, including quantitative real-time polymerase chain reaction (qRT-PCR) and enzyme-linked immunosorbent assay (ELISA).

local (intratympanic or intracochlear) application of DEX significantly rescues hearing loss ${ }^{11,20-27}$. Although reports in humans indicated the efficacy of DEX in intracochlear disorders, the mechanisms of action and timing of treatment have not been well established. Here, we investigated how noise trauma affects GR expression, corticosterone levels, and inflammatory responses. We also assessed the effects of the timing of DEX treatment for noise-induced hearing loss.

\section{Results}

Hair cell survival after noise exposure. Healthy CBA/J male mice were randomly assigned to two groups according to the noise exposure level: a transient threshold shift (TTS) group and a permanent threshold shift (PTS) group. To examine whether TTS or PTS causes hair cell loss/survival in the cochlea, whole-mount preparations of the auditory epithelium were stained with antibodies against myosin VIIa (red, hair cells) and phalloidin-FITC (green, F-actin) at 7 days after noise exposure as the schedule of the experiments is shown in Fig. 1. $\mathrm{OHCs}$ were more noticeably destroyed in the middle and basal turns of the cochlea in the PTS group (Fig. 2B1-B3) compared to the TTS group (Fig. 2A1-A3). Quantitative analysis of hair cell survival showed that PTS induced a significantly lower level of OHC survival (apex, middle, and basal turns, $\mathrm{p}<0.05$ ) compared to TTS (Fig. 2D). Loss of $\mathrm{OHC}$ was more prominent in the basal and middle turns compared to the apex turn in the PTS group (Fig. 2B2,B3,D). There was no significant difference in inner hair cell loss between TTS and PTS (Fig. 2C).

Expression of glucocorticoid receptor in the normal cochlea. GCs represent the only clinically proven treatment for various otological disorders and are known to exert their effects through GRs. Here, we determined which regions in the cochlea express GR. Immunohistochemistry for GR (labeled in red; Fig. 3) revealed wide expression of GR in the cochlea, specifically in the stria vascularis, hair cells, limbus, spiral ligament, and cochlear nerve (Fig. 3). The immunostaining results indicated that GR are significantly expressed in the whole cochlea under normal conditions.

Changes in GR mRNA expression. We next evaluated the impact of GR expression in the cochlea after noise exposure by treatment with the synthetic GC, DEX. Eight-week-old CBA/J male mice were randomly assigned to one of five groups: 1) normal; 2) noise exposure (TTS or PTS); and three DEX groups. Two DEX injections were administered at different time points: (1) pre-noise group (Pre-TTS/PTS), injections at 1 day before and immediately prior to noise exposure; (2) post-noise group (Post-TTS/PTS), injections immediately after and 1 day after noise exposure; and (3) pre\&post-noise group (Pre\&Post-TTS/PTS), injections 1 day before and 1 day after noise exposure (Fig. 1). Cochlear samples were collected at 1 and 3 days after noise exposure and analyzed for GR mRNA expression. As shown in Fig. 4A, TTS induced a significant decrease in GR mRNA expression at 1 day (normal vs. 1 day TTS-only, $\mathrm{P}=0.0002$ ) and 3 days (normal vs. 1 day TTS-only, $\mathrm{P}<0.0001$ ) after noise exposure. All DEX-administered subgroups (pre-noise, post-noise, and pre\&post-noise) showed a significant decrease in GR expression in comparison to normal mice, as well as TTS-only animals (Tukey's multiple comparison test, $\mathrm{P}<0.05$ ) at 1 day after noise exposure. The lower level of GR expression was maintained at 3 days after noise exposure in post- and pre\&post-DEX mice compared to the TTS-only group (Fig. 4A). The PTS group also showed a significant decrease in GR expression compared to normal mice at both 1 day and 3 days after noise exposure. Interestingly, the DEX-administered subgroups (pre-noise, post-noise, and pre\&post-noise) showed significant decreases in GR expression compared to normal animals; however, no significant changes were found in the DEX-administered subgroups compared to the PTS-only group (Fig. 4B), suggesting that GR expression induced by PTS decreased further than that induced by TTS, and thus diminished differences between PTS-only and PTS + DEX (all three subgroups). These data indicated that GR mRNA expression is significantly affected by noise exposure and DEX administration and that post and pre + post DEX treatments are comparable in GC receptor expression level. 


\section{TTS}
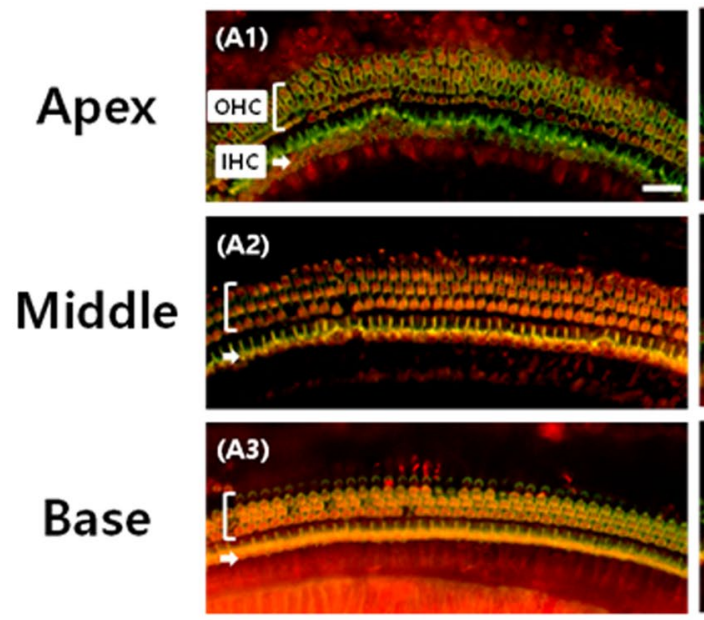

C

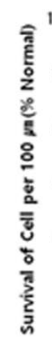

IHC

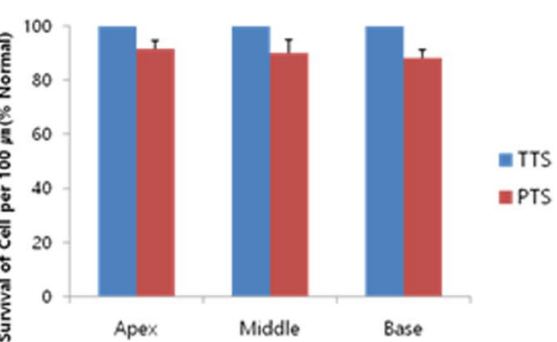

PTS
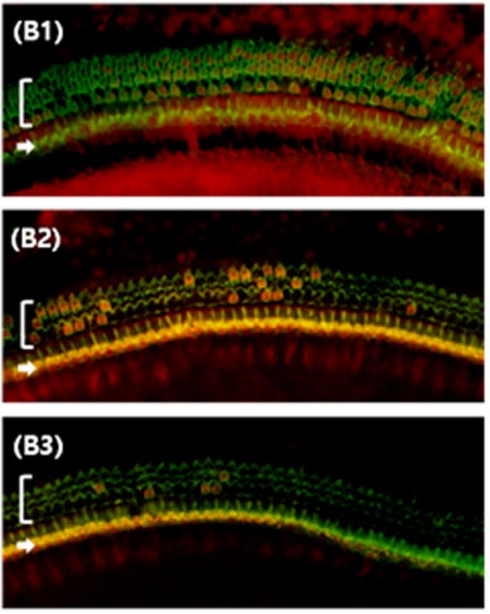

D

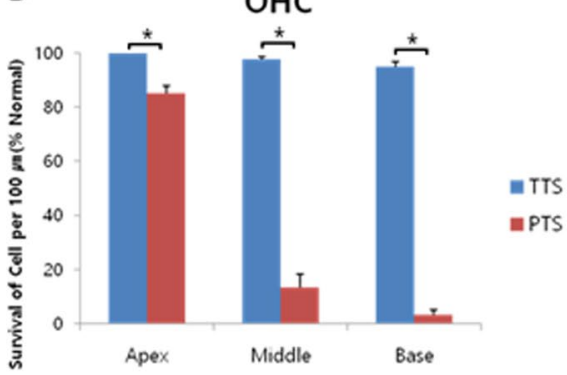

Figure 2. Whole-mount preparations of the auditory epithelium in the TTS (A1-A3) and PTS (B1-B3) groups. Tissues were stained for myosin VIIa (red) to visualize the hair cells and then photographed using epifluorescence. $(\mathbf{A}, \mathbf{B})$ Outer hair cells were more noticeably destroyed on the middle and basal turns of the cochlea in the PTS group (B1-B3) compared to the TTS group (A1-A3). Scale bar: $30 \mu \mathrm{m}$. (C,D) Quantitative analysis of hair cell survival on IHCs (C) and OHCs (D): apex, middle, and basal turns. TTS, transient threshold shift; PTS, permanent threshold shift; A1 and B1, apical turn; A2 and B2, middle turn; A3 and B3, basal turn; OHC, outer hair cell; IHC, inner hair cell. All graphs represent mean \pm S.E.M. $\mathrm{n}=3$ each group. ${ }^{*} \mathrm{p}<0.05$. Unpaired T-test.

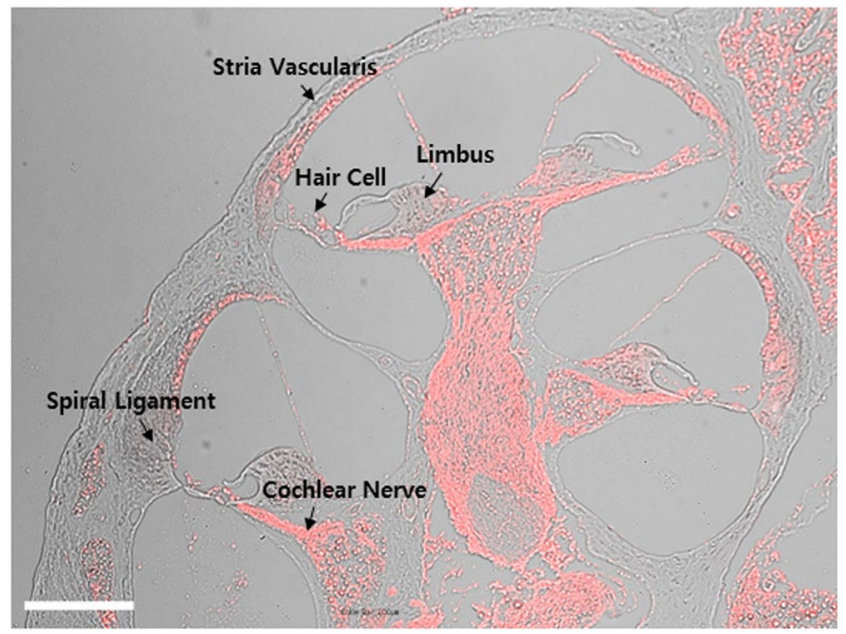

Figure 3. Localization of glucocorticoid receptors (GRs) in the mouse cochlea. Immunohistochemical analysis indicated that GRs (red) were widely expressed in the cochlea, including the stria vascularis, hair cells, limbus, spiral ligament, and cochlear nerve. 
A

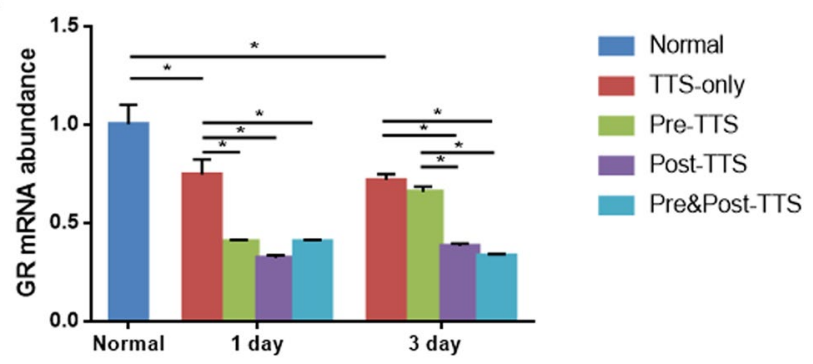

B

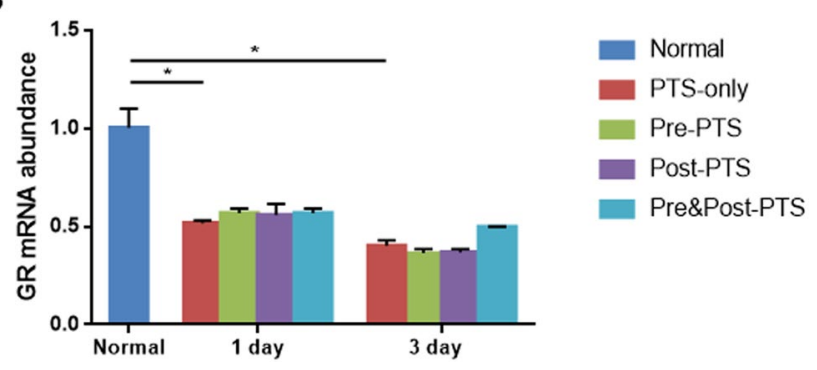

Figure 4. GR mRNA expression following noise exposure. (A) In the TTS group, GR mRNA expression was significantly decreased at 1 day after noise exposure compared to the untreated group. All three DEX-treated groups (pre-noise, post-noise, and pre\&post-noise) showed significantly decreased GR mRNA expression at 1 day after noise treatment, and the change was maintained in the post-noise and pre\&post-noise treatment groups at 3 days after noise exposure. (B) In the PTS treatment group, GR mRNA expression was significantly decreased after noise exposure, regardless of DEX treatment, at both days 1 and 3. All graphs represent mean \pm S.E.M. $\mathrm{n}=3$ each group, total 54 mice were used. One-way ANOVA and Tukey's multiple comparisons test. $* \mathrm{p}<0.05$.

Auditory brainstem response (ABR) threshold shifts. To evaluate how noise exposure changes the hearing threshold in different groups, the ABR thresholds at $4,8,16$, and $32 \mathrm{kHz}$, and click sounds were measured at four time points as indicated in Fig. 1: before noise exposure (baseline); immediately after; 1, 3, and 7 days following noise exposure. As shown in Fig. 5A, the ABR threshold shift significantly increased immediately after noise exposure and showed a declining pattern over the next 7 days, indicating that the animals recovered from TTS over time. Pre\&post DEX treatment significantly improved threshold shift as compared to TTS-only at $8 \mathrm{kHz}$ (Two-way Repeated Measures ANOVA; Tukey's multiple comparisons test; $n=6$ each group; 0 day, $p=0.0088$; and 1 day, $\mathrm{p}=0.0266), 16 \mathrm{kHz}(1$ day, $\mathrm{p}=0.0056), 32 \mathrm{kHz}(0 \mathrm{hr}, \mathrm{p}=0.0212)$, and click ( 1 day, $\mathrm{p}=0.0293)$. Moreover, pre\&post treatment was significantly different from the pre treatment at $4 \mathrm{kHz}$ ( 3 day, $\mathrm{p}=0.0422$ ), $8 \mathrm{kHz}$ ( 3 day, $\mathrm{p}=0.0266$ ), and $16 \mathrm{kHz}$ ( 1 day, $\mathrm{p}=0.0185$ ). Threshold shift at $16 \mathrm{kHz}$ (Two-way Repeated Measures ANOVA; Tukey's multiple comparisons test; $\mathrm{n}=6$ each group; main effect time, $\left.\mathrm{F}_{(3,15)}=9.441, \mathrm{p}=0.0009\right)$ also revealed that pre\&post DEX significantly improved hearing loss at 1 day post noise exposure as compared to all other groups (pre\&post vs. pre, $p=0.0185$; pre\&post vs. post, $p=0.019$ ). No differences in $A B R$ threshold shifts were observed in DEX treatment groups compared to the PTS-only group (Fig. 5B). Collectively, these data support that the timing of DEX administration differentially effects functional recovery after noise trauma.

Circulating corticosterone level with noise exposure and DEX administration. To test circulating levels of corticosterone, serum samples were collected from mice before (normal) or after (1 day and 3 days) noise exposure and assayed for corticosterone. The corticosterone levels are shown in Fig. 6. TTS induced a significant increase in serum corticosterone level compared to normal mice at 1 and 3 days after noise exposure (Fig. 6A). DEX subgroups did not show a significant increase in the corticosterone level compared to normal mice at 1 day after noise exposure, indicating that DEX treatments prevented the increase in corticosterone level after noise trauma (Fig. 6A). The post-DEX group (post-TTS) showed a similar corticosterone level to the TTX-only group at 3 days following noise exposure, whereas the corticosterone levels in the other DEX groups (pre- and pre\&post-TTS) remained decreased compared to the TTS-only group at 3 days after noise trauma (Fig. 6A). PTS also caused significant increases in circulating corticosterone levels compared to normal animals at 1 and 3 days after noise exposure (Fig. 6B). The DEX subgroups showed a similar pattern in terms of PTS as observed in the TTS experiments (Fig. 6A). These observations suggested that serum corticosterone levels are significantly affected by noise exposure and DEX administration.

Changes in inflammatory cytokines. To test cytokine levels in the cochlea, we collected tissue samples from the five groups described above at 1 day after noise trauma and performed quantitative real-time polymerase chain reaction (qRT-PCR) assays for heme oxygenase-1 (HO-1), interleukin-1 $\beta$ (IL-1 $\beta$ ), tumor necrosis factor- $\alpha$ (TNF- $\alpha$ ), and interleukin-6 (IL-6). Figure 7 shows that the expression levels of HO-1 and the proinflammatory 
A

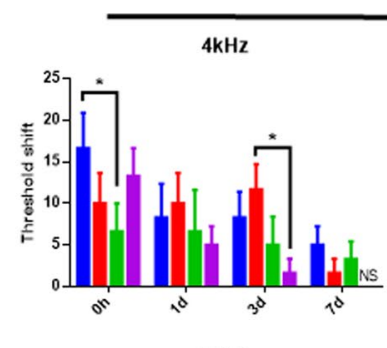

$8 \mathrm{kHz}$
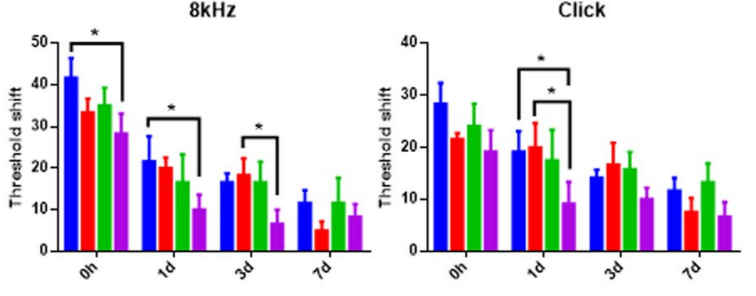

$16 \mathrm{kHz}$
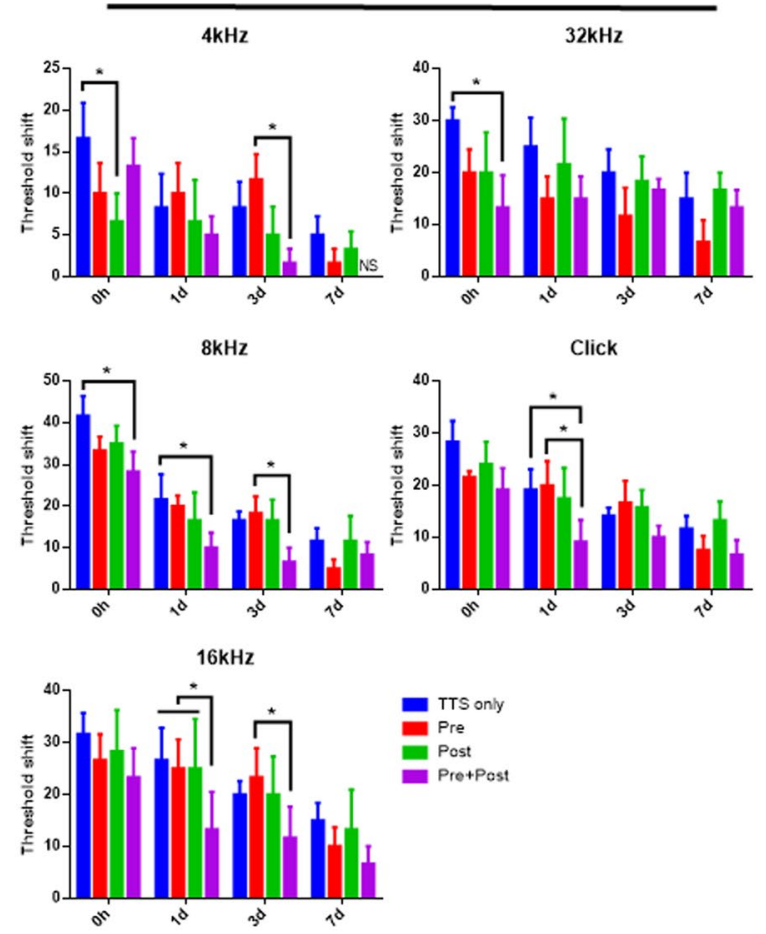

B

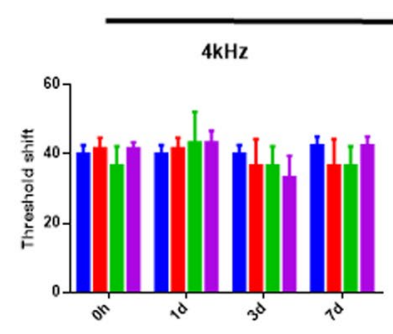

PTS

$8 \mathrm{kHz}$
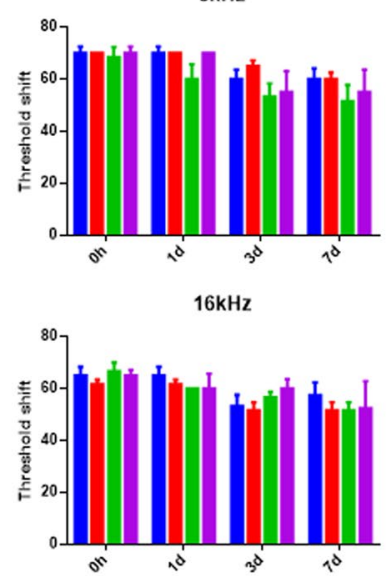
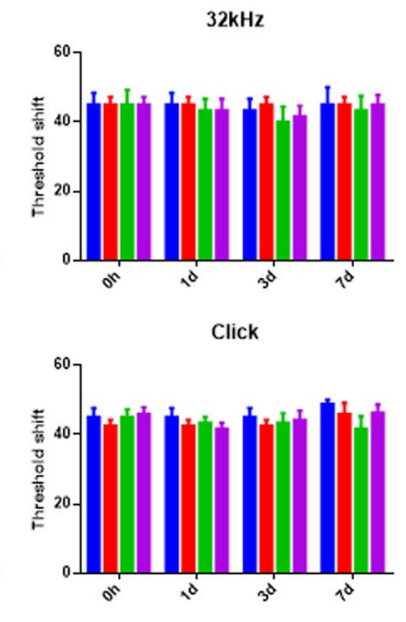

Click

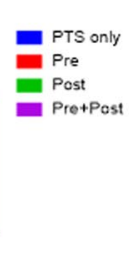

Figure 5. ABR threshold shifts with or without DEX administration. ABR thresholds were measured at five time points: prior to, immediately after, and 1,3 and 7 days after noise exposure. (A) In the TTS group, increased ABR threshold shifts were observed in all groups after noise exposure and gradually decreased over time. ABR threshold shifts were significantly reduced in the pre\&post DEX group compared to the TTS-only group at some frequencies and click. (B) PTS induced prolonged ABR threshold shifts at all time points until 7 days after noise exposure. There were no significant differences among treatment groups. All graphs represent mean \pm S.E.M. Two-way repeated measures ANOVA and Tukey's multiple comparisons test $(\mathrm{n}=6$ each group, total 48 mice were used). ${ }^{*} \mathrm{p}<0.05$.

cytokines, IL-1 $\beta$, TNF- $\alpha$, and IL-6, were significantly increased in TTS- and PTS-treated groups compared to normal animals. DEX administration significantly decreased the expression levels of the cytokines and HO-1 compared to the TTS- and PTS-only groups. No significant differences were found among the three DEX treatment groups. These observations suggested that exposure to noise induces a local cochlear inflammatory response, which can be ameliorated by DEX administration.

\section{Discussion}

The results of this study showed that noise trauma decreased cochlear GR expression (Fig. 4), and increased both serum corticosterone level (Fig. 6) and cochlear cytokine expression (Fig. 7). Treatment with the synthetic GC, DEX, further decreased the GR mRNA level in the cochlea (Fig. 4), and prevented increases in serum corticosterone and cytokine levels (Figs 6 and 7). This study also provided insight into the potential mechanism underlying the effectiveness of DEX for rescuing noise-induced hearing loss. Threshold shift at $16 \mathrm{kHz}$ revealed that pre\&post DEX significantly improved hearing loss at $1 \mathrm{~d}$ post noise trauma as compared to all other groups (Fig. 5).

$\mathrm{GC}$ is widely used for the treatment of inner ear disease, exerting its effects by binding to its receptor, $\mathrm{GR}^{2}$. Therefore, the expression of GR mediates the effects of $\mathrm{GC}^{28}$. GRs are highly expressed throughout the inner ear, including the stria vascularis, inner hair cells, OHCs, and spiral ligament of the cochlea and cochlear nerve $\mathrm{e}^{13,14}$. The GR mRNA expression level was reported to be significantly decreased following acoustic trauma in the coch$\mathrm{lea}^{29}$ as well as in the organ of Corti ${ }^{30}$. Terunuma et al. reported that GR mRNA expression was significantly decreased following acoustic trauma ${ }^{29}$. Mori et al. reported that compound action potentials (CAPs) at a threshold of $5-8 \mathrm{kHz}$ were significantly elevated when the GR antagonist, mifepristone, was administered following exposure to noise with a sound pressure level (SPL) of $120 \mathrm{~dB}^{31}$. The ABR threshold shift was found to be significantly decreased by pretreatment with the corticosteroid, methylprednisolone ${ }^{32}$. The GR antagonist, RU486, and the GC synthesis inhibitor, metyrapone, significantly increased the ABR threshold shift ${ }^{32,33}$. In the present study, we confirmed that noise trauma, including TTS and PTS, significantly decreased GR mRNA expression in the cochlea (Fig. 4).

The results shown in Fig. 4 raise the question of how TTS and PTS differentially affect local cochlear GR mRNA expression. GR expression was further diminished by PTS compared to TTS at 1 day $(0.5185 \pm 0.0074$ vs. $0.7468 \pm 0.0441, P=0.0070$, unpaired $t$ test $)$ and 3 days $(0.4035 \pm 0.0154$ vs. $0.7180 \pm 0.0177, P=0.0002$, unpaired $t$ test), suggesting that GR expression may be inversely correlated to the degree of stress. The expression levels of 


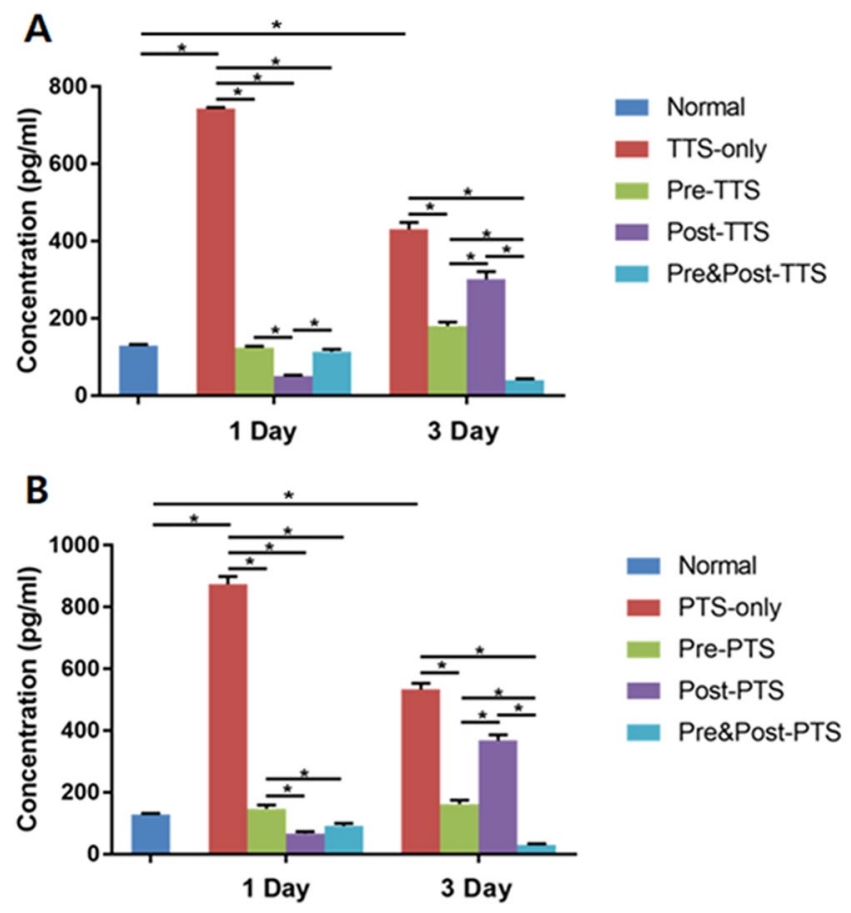

Figure 6. Serum corticosterone levels at 1 and 3 days after noise exposure. Noise exposure (both TTS and PTS) significantly increased the circulating corticosterone level at early (1 day) and late (3 days) acute phases after noise exposure. DEX administration maintained a low circulating level of corticosterone after noise trauma. Interestingly, the post-DEX group showed significantly lower levels of corticosterone compared to the pre-DEX and pre\&post-DEX groups in the early acute phase (1 day). At the late acute phase (3 days), post-DEX showed significantly increased serum corticosterone levels in comparison to the other DEX treatment groups. All graphs represent mean \pm S.E.M. One-way ANOVA and Tukey's multiple comparisons test $(\mathrm{n}=3$ each group, total 54 mice were used). $* \mathrm{p}<0.05$.

the GR isoform, GR $\alpha$, were shown to be negatively correlated with the number/degree of stressful events experienced in a clinical study of posttraumatic stress disorder (PTSD) ${ }^{34}$. Although DEX partially rescued TTS-induced hearing impairment (Fig. 5A), it was ineffective in the PTS group (Fig. 5B). These observations suggested that the dose and/or timing of DEX administration were insufficient to rescue the permanent cell damage (as shown in Fig. 2) induced by intense acoustic trauma (PTS).

The noise trauma increased the endogenous circulating corticosterone level (Fig. 6), with a subsequent decrease in cochlear GR mRNA expression (Fig. 4). The magnitude of hearing loss induced by noise trauma was decreased by pre\&post treatment with the GR agonist, DEX (Fig. 5), which was accompanied by diminished cochlear GR mRNA expression (Fig. 4). Tahera et al. reported that treatment with the GR antagonist, RU486, and the GC synthesis inhibitor, metyrapone, prior to acoustic trauma increased GR mRNA expression in the cochlea ${ }^{33}$. In contrast, Helling's group reported that pretreatment with the GR agonist, DEX, significantly increased GR expression compared to a noise exposure group in a different region of the cochlea, the spiral ligament ${ }^{25}$. Further studies to investigate how acoustic trauma and/or DEX treatment affect GR expression in specific subregions of the cochlea are required.

Acoustic trauma is one of the stressors that raise circulating corticosterone level ${ }^{35}$ by stimulating the hypothalamic-pituitary-adrenal axis ${ }^{33}$. In this study, serum corticosterone levels were increased in both TTS and PTS groups after noise exposure (Fig. 6). The serum corticosterone level was as low as in normal mice at 1 day in all DEX treatment groups, and this low level was maintained until 3 days only in the pre\&post DEX treatment group (Fig. 6), consistent with the improved hearing function in the same group (Fig. 5). It is possible that exogenous DEX administration plays a role in maintaining homeostasis by acting as an endogenous source of corticosterone in the cochlea. The decrease in GR mRNA level may be due to feedback inhibition by increased endogenous stress hormone, corticosterone after acoustic stress as shown in Fig. 6. We speculate that the increase may be an acute response after noise stress and maintained at a high level for at least 3 days after the trauma. The small nitric oxide (NO) molecule could be responsible for the increase in GR mRNA expression after noise stress. Noise exposure significantly increases the NO production in the cochlea, which may degrade proteins by ubiquitination and/or proteasomal activity of the cytochrome $\mathrm{P} 450^{36}$. Another potential mechanism may be differences in the phosphorylation status of the GR mRNA. The mouse GR contains eight phosphorylation sites, so that the phosphorylation status of GR may pre-determine the GR protein turnover and local GR degradation after stress ${ }^{37-40}$

While we found a diminished GR expression after noise trauma and a further downregulation of GR in DEX treatment groups (Fig. 4) from the whole cochlear extracts, Heinrich et al. reported DEX treatment restored GR 
A

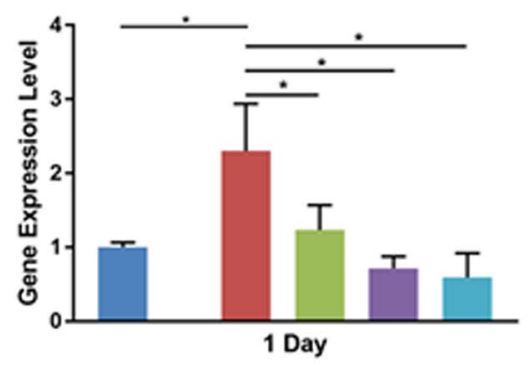

TNF- $\alpha$

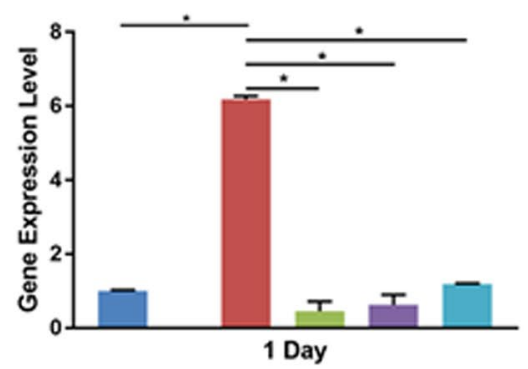

B

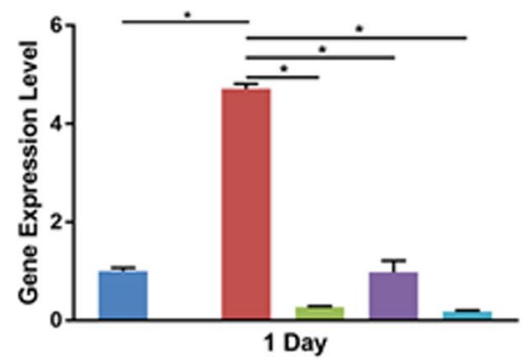

TNF- $\alpha$

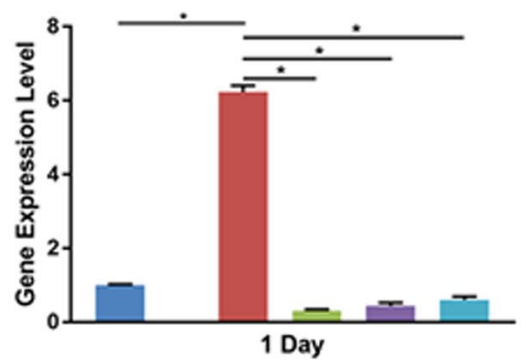

IL-1 $\beta$
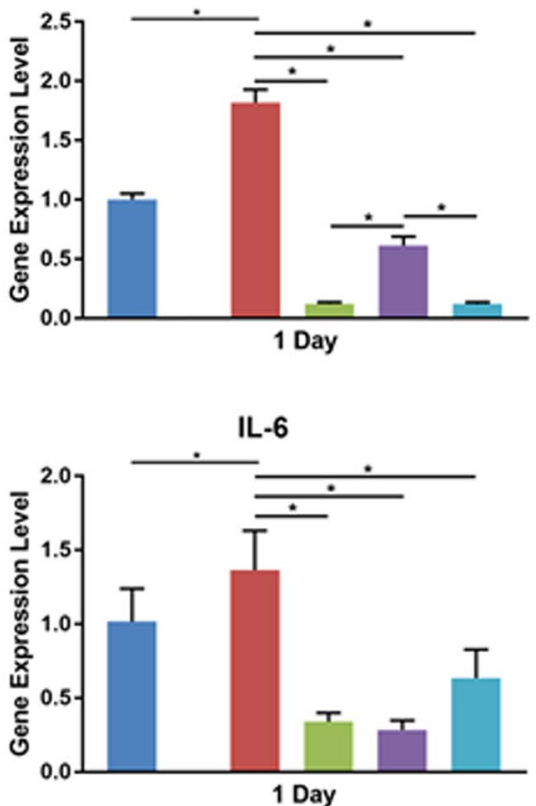

IL-1 $\beta$
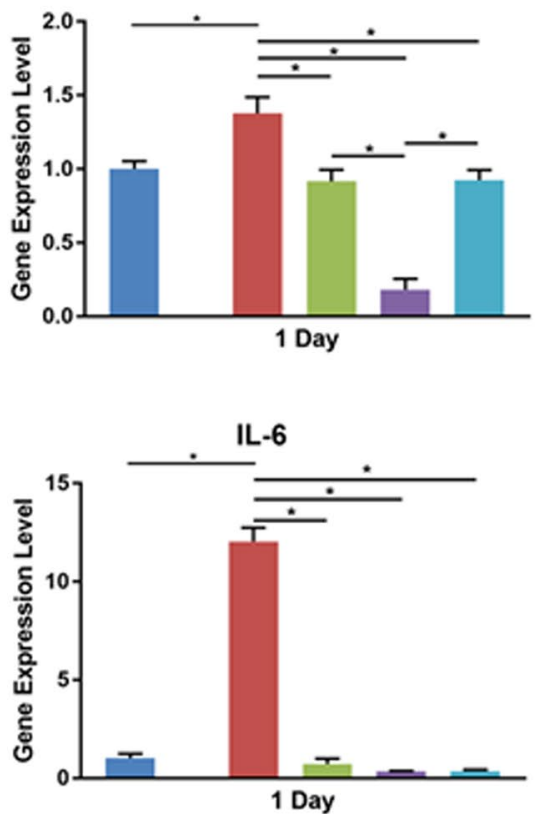

Normal

PTS-only

Pre-PTS

Post-PTS

Pre\&Post-PTS

Figure 7. Inflammatory cytokine expression in the cochlea. Both TTS (7A) and PTS (7B) significantly increased expression levels of heme oxygenase-1(HO-1), interleukin-1 $\beta$ (IL-1 $\beta$ ), tumor necrosis factor- $\alpha$ (TNF$\alpha$ ), and interleukin-6 (IL-6) in the cochlea compared to non-treated animals. IL-1 $\beta$ was significantly decreased in the pre- and pre\&post-TTS groups compared to the post-TTS group, and in the post-PTS group compared to the pre- and pre\&post-PTS groups. All graphs represent mean \pm S.E.M. *One-way ANOVA and Tukey's multiple comparisons test ( $\mathrm{n}=3$ each group, total 30 mice were used). ${ }^{*} \mathrm{p}<0.05$.

intensity as compared to noise trauma per se in Guinea pig. Others have reported contradicting outcomes by showing that DEX down-regulates GR mRNA expression in rat hepatoma culture (HTC) cells ${ }^{41}$. They presented that glucocorticoid-treatment for $24-48 \mathrm{hr}$ resulted in a down-regulation of cellular GR mRNA levels in both HTC cells and in rat liver in $v i v o^{41}$. One of the important observations was the transient downregulation of GR, since the GR mRNA level was restored after $72 \mathrm{hr}^{41}$. More interestingly, an initial increase of GR mRNA was observed before the down-regulation occurred. These reports indicate that GR mRNA expression is affected in a time-sensitive manner after DEX treatment, and its response may be variable depending on tissue- and/or 
cell-types. Steroid hormone receptors are well known to function as transcription factors mediating the biological effects of steroids by regulating gene expression. However, it is also reported that the receptors are regulated both by transcriptional and posttranslational mechanisms ${ }^{42}$. This implies that measuring the GR protein levels along with mRNA expression may be important to access DEX-induced GR alterations. Therefore, in-depth studies on GC and GR mechanisms are further needed.

Cytokines, including IL-1 $\beta$, TNF- $\alpha$, and IL-6, were maintained at low levels after DEX administration compared to the noise exposure-only group (Fig. 7). These proinflammatory cytokines have been suggested to cause brain damage similar to free radicals ${ }^{43}$. Several studies reported the presence of inflammatory cells and cytokines in the cochlea following acoustic trauma ${ }^{44,45}$ with a significantly increased ABR threshold shift ${ }^{33}$.

A key question is how GC and GR signaling modulates inflammatory cytokines. GR signaling is known to be mediated by two pathways: a classical genomic (DNA binding-dependent) pathway and a non-genomic (DNA binding-independent) pathway. The classical genomic pathway modulates the expression of target genes by binding to specific DNA sequences within the nucleus after GC binds to the GR ${ }^{6,46,47}$. Some of the GRs associate with transcription factors, such as AP-1 and NFKB (essential molecules in upregulation of the inflammatory response), which is the pathway by which GCs exert the majority of their antiinflammatory effects ${ }^{48}$. The non-genomic (DNA binding-independent) pathway can modulate gene expression without binding to hormone response elements (HREs), and is also considered to underlie a number of immunosuppressive and antiinflammatory acti ons o $^{2,33,49,50}$.

Previous studies have used various doses of DEX. For instance, Weichhart et al. utilized DEX intraperitoneally at $600 \mu \mathrm{g}(\sim 30 \mathrm{mg} / \mathrm{kg})$ to study the immune suppressive effects of DEX after LPS-induced inflammation in a mouse model ${ }^{51}$. Tuckermann et al. injected DEX intraperitoneally at a dose of $25 \mu \mathrm{g}$ and subsequently continued with $20 \mathrm{mg} / \mathrm{L}$ dexamethasone (drinking water) in C57BL/6 and Balb/c mice ${ }^{52}$. Sadikot et al. used DEX in a range of $0.3 \mathrm{ug} / \mathrm{g}$ to $10 \mathrm{ug} / \mathrm{g}$ in transgenic reporter mice (HLL mice) to investigate DEX treatment on NF-kappa B activa$\operatorname{tion}^{53}$. These reports support that our usage of DEX concentration, $20 \mathrm{ug} / \mathrm{g}(=20 \mathrm{mg} / \mathrm{kg})$, is not far from the doses generally utilized in pre-clinical studies. However, regardless the conventional-dose of DEX in animal studies, our DEX dose far exceeds the dose used in humans. Therefore, further preclinical studies with DEX concentrations that can be reasonably translated to human dose seem necessary.

In summary, the results of the present study indicated that serum corticosterone level and GR expression are important for maintenance of cochlear homeostasis. In addition, DEX administration may be recommended before and after acoustic trauma, especially in soldiers before leaving for a battlefield, which may minimize noise-induced hearing loss by regulating GR mRNA and immune responses.

\section{Methods}

Animals. All animal experiments were approved by Chungnam National University, Institutional Animal Care and Use Committee (CNU00936). CBA/J male mice, aged 8 weeks, weighing 25-30 g, were confirmed to have normal hearing prior to noise exposure, were used in this study. Healthy mice were randomly divided into two groups according to the noise exposure level: a transient threshold shift (TTS) group and a permanent threshold shift (PTS) group. Each group was further divided into four subgroups according to the timing of DEX administration: noise exposure only, pre-noise, post-noise, and pre\&post-noise. The experimental animals were used for time point studies on days 1, 3, and 7 following exposure. To avoid any impact of circadian rhythm on hormone levels, serum samples were collected at the same time of day (between 12:00 and 14:00). The schedule of the experiments is shown schematically in Fig. 1.

Noise exposure. In the TTS groups, animals were exposed to free-field broadband noise $(250 \mathrm{~Hz}-8 \mathrm{kHz})$ for 20 minutes at an intensity of 116 decibels $(\mathrm{dB})$ SPL following previously-published protocols ${ }^{20}$. In the PTS groups, free-field broadband noise $(2-8 \mathrm{kHz})$ was applied for 2 hours at an intensity of $116 \mathrm{~dB}$ SPL in an acoustically insulated reverberation chamber as described previously ${ }^{20,54}$. The noise signals were routed through a computer and an amplifier (INTER-M R300 Plus power amplifier; Canford Audio PLC, Washington, UK) to a loud speaker (ElectroVoice DH1A-WP; Sonic Electronix Inc., Sylmar, CA). The noise level was measured using a sound level meter (B\&K type 2250; Brüel \& Kjaer, Naerum, Denmark), sound calibrator (B\&K type 4231; Brüel \& Kjaer), and condenser microphone (B\&K type 4189; Brüel \& Kjaer).

Dexamethasone injection. DEX ( $5 \mathrm{mg} / \mathrm{mL}$ dexamethasone sodium phosphate; Huons, Sungnam, Korea) was injected intraperitoneally at a dose of $20 \mu \mathrm{g} / \mathrm{g}$. The pre-noise subgroups were injected with DEX at 1 day before and immediately before noise exposure. The post-noise subgroups were administered DEX immediately after and 1 day after noise exposure, and the pre\&post-noise subgroups were administered DEX at 1 day before and 1 day after noise exposure. Noise exposure-only subgroups did not receive DEX injection.

Auditory brainstem response. ABR thresholds at frequencies between 4 and $32 \mathrm{kHz}$, and click sounds, were obtained separately from both ears as described previously ${ }^{20}$. ABRs were recorded prior to noise exposure, immediately after, and 1, 3 and 7 days after noise exposure. The TDT System-3 (Tucker Davis Technologies, Gainesville, FL) hardware and software were used to obtain the ABRs. The stimuli were computer-generated tone pips.

The animals were anesthetized with intramuscular injection of zolazepam $\mathrm{HCl} 40 \mathrm{mg} / \mathrm{kg}$ (Zoletil, Virbac Animal Health, Carros, France) and xylazine $10 \mathrm{mg} / \mathrm{kg}$ (Rompun, Bayer Animal Health, Monheim, Germany) ${ }^{20}$. Subcutaneous needle electrodes were placed around the skull vertex and both infraauricular areas. Tone bursts, with a duration of $4 \mathrm{~ms}$ and rise-fall time of $1 \mathrm{~ms}$ at frequencies of $4,8,16,32 \mathrm{kHz}$, were used, in addition to clicks. The sound intensity was varied in $10-\mathrm{dB}$ increments for the tone burst sounds and in $5-\mathrm{dB}$ increments for the click and tone burst sounds close to the threshold. The contralateral ear was not masked because the stimuli were 


\begin{tabular}{|c|c|c|}
\hline Primer name & & Sequence $\left(5^{\prime}-3^{\prime}\right)$ \\
\hline \multirow[t]{2}{*}{ GAPDH } & Forward & 5'-TGTGTCCGTCGTGGATCTGA-3' \\
\hline & Reverse & 5'-CCTGCTTCACCACCTTCTTGAT-3' \\
\hline \multirow[t]{2}{*}{ GR } & Forward & 5'-CCCAAGAGTTCAACACCTGC-3' \\
\hline & Reverse & 5'-AAACTCCTTCTCTGTCGGGG-3' \\
\hline \multirow[t]{2}{*}{ HO-1 } & Forward & 5'-CCCACCAAGTTCAAACAGTCT-3' \\
\hline & Reverse & 5'-AGGAAGGGGGTCTTAGCCTC-3' \\
\hline \multirow[t]{2}{*}{ IL-1 $1 \beta$} & Reverse & 5'-TCTTTGAAGTTGACGGACCC-3' \\
\hline & Reverse & 5'-TGAGTGATACTGCCTGCCTG-3' \\
\hline \multirow[t]{2}{*}{ TNF- $\alpha$} & Forward & 5'-CTGAGGTCAATCTGCCCAAGTAC-3' \\
\hline & Reverse & 5'-CTTCACAGAGCAATGACTCCAAAG-3' \\
\hline \multirow[t]{2}{*}{ IL-6 } & Forward & 5'-TCGTGGAAATGAGAAAAGAGTTG-3' \\
\hline & Reverse & 5'-AGTGCATCATCGTTGTTCATACA-3' \\
\hline
\end{tabular}

Table 1. Primer sequences used in this study for quantitative RT-PCR.

transmitted through a sealed earphone. The waveforms were analyzed using a custom program (BioSig RP, ver. 4.4.1; Tucker Davis Technologies) with the researcher blinded to the treatment group. Threshold was defined as the lowest stimulus intensity to evoke a wave III response $>0.2 \mu \mathrm{V}$.

Quantitative real-time polymerase chain reaction. Animals were sacrificed at either 1 or 3 days after the surgical procedures and qRT-PCR was performed to evaluate the expression of GR and degree of inflammation. HO-1, IL-1 $\beta$, TNF- $\alpha$, and IL-6 were measured as indicators of inflammatory response ${ }^{20,54}$.

Dissected cochleae were ground in $1 \mathrm{ml}$ of TRIzol reagent (Invitrogen, Carlsbad, CA), and $200 \mu \mathrm{l}$ of chloroform was added followed by centrifugation at 13,000 rpm for 15 minutes. About $450 \mu$ of supernatant was transferred to a fresh tube and an equal volume of isopropanol was added, shaken for 5 minutes, and centrifuged at $13,000 \mathrm{rpm}$ for 15 minutes. The resulting pellet was resuspended in $1 \mathrm{ml}$ of $80 \%$ ethanol in DEPC-treated water and centrifuged at 13,000 rpm for 15 minutes. The same procedure was performed one more time and the pellet was then washed repeatedly with $100 \%$ ethanol. RNA was dissolved in $20 \mu$ of RNase-free water. The purified RNA was quantified using a Nanodrop instrument (NanoDrop Technologies Inc., Wilmington, DE) by measuring the absorbance at $260 \mathrm{~nm}$. A total of $13 \mu \mathrm{l}$ of RNA $(2 \mu \mathrm{g}$ each) with oligo-dT primer and DEPC-treated water was pre-denatured for 10 minutes at $65^{\circ} \mathrm{C}$; $4 \mu \mathrm{l}$ of $5 \times$ reaction buffer, $2 \mu \mathrm{l}$ of dNTP, $0.5 \mu \mathrm{l}$ of RNase inhibitor, and $0.5 \mu$ l of reverse transcriptase were added and reverse-transcribed for 1 hour at $50^{\circ} \mathrm{C}$ and 5 minutes at $85^{\circ} \mathrm{C}$ with a cDNA Synthesis Kit (Roche, Indianapolis, IN). Real-time reverse transcription was performed according to the manufacturer's protocol with SYBR Green (Invitrogen, Grand Island, NY). Comparative quantification of GR, HO-1, IL-1 $\beta$, TNF- $\alpha$, and IL-6 mRNA was performed using the cycle threshold method. qRT-PCR was performed three times for each sample. Details of the primers used in PCR to detect GR, HO-1, IL-1 $\beta$, TNF- $\alpha$, and IL-6 are presented in Table 1.

Enzyme-linked immunosorbent assay. After sacrificing the animals, blood samples were collected in heparinized tubes at the same time of day to minimize the circadian fluctuation of corticosterone level (between 12:00 and 14:00). Plasma was separated by spinning at 3,000 rpm at room temperature, and was then immediately stored at $-20^{\circ} \mathrm{C}$ until corticosterone assay. The serum corticosterone level was determined using an ELISA kit (Corticosterone ELISA kit, ADI-900-097; Enzo Life Sciences, Farmingdale, NY) with a sensitivity of $5 \mathrm{pg} / \mathrm{ml}$.

Tissue preparation and immunohistochemistry. The animals were sacrificed before (Fig. 3 ) or 7 days after noise exposure (Fig. 2). Cochlear tissues were obtained to localize glucocorticoid receptors (Fig. 3) and assess the survival of hair cells and nerve fibers (Fig. 2). Tissues were fixed in $4 \%$ paraformaldehyde in phosphate-buffered saline (PBS) for 1 hour at room temperature. After removal of the cochlear bony walls and lateral wall tissues, the remaining cochlear tissues were prepared for immunostaining. Tissues were permeated with $0.3 \%$ Triton X-100 (Sigma-Aldrich, St. Louis, MO) for 10 minutes, blocked in 5\% normal goat serum (Vector Laboratories, Burlingame, CA) for 30 minutes, and then incubated with mouse anti-GR primary antibody (Santa Cruz Biotechnology, Dallas, TX) - Alexa 594 (Invitrogen-Molecular Probes, Eugene, OR) or rabbit anti-myosin VIIa primary antibody (Proteus BioSciences, Ramona, CA) - Alexa Fluor 488 Phalloidin (A12379; Invitrogen-Molecular Probes, Eugene, OR) at a concentration of 1:200 in blocking solution overnight at $4{ }^{\circ} \mathrm{C}$. After rinsing in PBS for 10 minutes, the tissues were incubated with the AlexaFluor 594 goat anti-rabbit secondary antibody (Molecular Probes) at a concentration of 1:200 in PBS for 30 minutes. After rinsing in PBS for 10 minutes, specimens were further dissected to separate individual cochlear turns, and mounted on glass slides using Crystalmount (Biomeda, Foster City, CA). The specimens were observed under an epifluorescence microscope (Zeiss Axio Scope A1; Zeiss, Oberkochen, Germany) with a digital camera. The timelines for all experiments are shown in Fig. 1.

Image processing and statistical analysis. Adjustment of image contrast, superimposition of images, and colorization of monochrome fluorescence images were performed using Adobe Photoshop (version 7.0; Adobe, San Jose, CA). Power analysis, using data from pilot studies and other experiments using CBA/J male 
mice, estimated sample size at three. Most groups had a larger sample size, with three as the minimum. One-way ANOVA was used for enzyme-linked immunosorbent assay and qRT-PCR. For ABR, a two-way repeated measures ANOVA coded for treatment and day/time was used. For hair cell counts, an unpaired Student's t-test was used. Group differences were considered significant at $\mathrm{p}<0.05$ in each case. All data presented in bar graphs are the mean \pm S.E.M. from multiple determinations.

Compliance with ethical standards. All experimental protocols were approved by Chungnam National University Institutional Animal Care and Use Committee. All animal care and use was conducted in accordance with the Guide for the Care and Use of Laboratory Animals.

\section{Data Availability}

All data generated or analyzed during this study are included in this published article.

\section{References}

1. Chrousos, G. P. \& Gold, P. W. The concepts of stress and stress system disorders. Overview of physical and behavioral homeostasis. Jama 267, 1244-1252 (1992).

2. Guyre, P. M. \& Munck, A. Glucocorticoids. (1998).

3. Frew, A. J. In Clinical Immunology (Fifth Edition) 1227-1235. e1221 (Elsevier, 2019).

4. Spies, C. M. et al. Glucocorticoids. 25, 891-900 (2011).

5. Alexiou, C. et al. Sudden sensorineural hearing loss: does application of glucocorticoids make sense? Archives of otolaryngology-head \& neck surgery 127, 253-258 (2001).

6. Meltser, I. \& Canlon, B. Protecting the auditory system with glucocorticoids. Hearing research 281, 47-55, https://doi.org/10.1016/j. heares.2011.06.003 (2011).

7. Barrs, D. M. Intratympanic corticosteroids for Meniere's disease and vertigo. Otolaryngologic clinics of North America 37, 955-972, v, https://doi.org/10.1016/j.otc.2004.03.004 (2004).

8. Rauch, S. D. Intratympanic steroids for sensorineural hearing loss. Otolaryngologic Clinics of North America 37, 1061-1074 (2004).

9. Westerlaken, B. O., de Kleine, E., van der Laan, B. \& Albers, F. The treatment of idiopathic sudden sensorineural hearing loss using pulse therapy: a prospective, randomized, double-blind clinical trial. The Laryngoscope 117, 684-690 (2007).

10. Siegel, L. The treatment of idiopathic sudden sensorineural hearing loss. Otolaryngologic Clinics of North America 8, 467 (1975)

11. Morawski, K., Telischi, F. F., Bohorquez, J., Niemczyk, K. J. O. \& Neurotology. Preventing hearing damage using topical dexamethasone during reversible cochlear ischemia: an animal model. 30, 851-857 (2009).

12. Shirwany, N. A., Seidman, M. D. \& Tang, W. Effect of transtympanic injection of steroids on cochlear blood flow, auditory sensitivity, and histology in the guinea pig. Am J Otol 19, 230-235 (1998).

13. Terakado, M., Kumagami, H. \& Takahashi, H. Distribution of glucocorticoid receptors and 11 beta-hydroxysteroid dehydrogenase isoforms in the rat inner ear. Hear Res 280, 148-156, https://doi.org/10.1016/j.heares.2011.05.006 (2011).

14. Shimazaki, T., Ichimiya, I., Suzuki, M. \& Mogi, G. Localization of glucocorticoid receptors in the murine inner ear. Ann Otol Rhinol Laryngol 111, 1133-1138, https://doi.org/10.1177/000348940211101213 (2002).

15. Rarey, K. E. \& Curtis, L. M. Receptors for glucocorticoids in the human inner ear. Otolaryngol Head Neck Surg 115, 38-41, https:// doi.org/10.1016/s0194-5998(96)70133-x (1996).

16. Bookout, A. L. et al. Anatomical profiling of nuclear receptor expression reveals a hierarchical transcriptional network. Cell 126, 789-799 (2006).

17. John, S. et al. Chromatin accessibility pre-determines glucocorticoid receptor binding patterns. Nature genetics 43, 264 (2011).

18. So, A. Y.-L., Chaivorapol, C., Bolton, E. C., Li, H. \& Yamamoto, K. R. Determinants of cell-and gene-specific transcriptional regulation by the glucocorticoid receptor. PLoS genetics 3, e94 (2007).

19. John, S. et al. Kinetic complexity of the global response to glucocorticoid receptor action. Endocrinology 150, 1766-1774 (2009)

20. Lyu, A. R. et al. Effects of dexamethasone on intracochlear inflammation and residual hearing after cochleostomy: A comparison of administration routes. PLoS One 13, e0195230, https://doi.org/10.1371/journal.pone.0195230 (2018).

21. Takemura, K. et al. Direct inner ear infusion of dexamethasone attenuates noise-induced trauma in guinea pig. 196, 58-68 (2004).

22. Bird, P. A., Murray, D. P., Zhang, M., Begg, E. J. J. O. \& Neurotology. Intratympanic versus intravenous delivery of dexamethasone and dexamethasone sodium phosphate to cochlear perilymph. 32, 933-936 (2011).

23. Eastwood, H. et al. Round window delivery of dexamethasone ameliorates local and remote hearing loss produced by cochlear implantation into the second turn of the guinea pig cochlea. 265, 25-29 (2010).

24. Eshraghi, A. A. et al. Local dexamethasone therapy conserves hearing in an animal model of electrode insertion trauma-induced hearing loss. 28, 842-849 (2007).

25. Heinrich, U. R., Strieth, S., Schmidtmann, I., Stauber, R. \& Helling, K. Dexamethasone prevents hearing loss by restoring glucocorticoid receptor expression in the guinea pig cochlea. Laryngoscope 126, E29-34, https://doi.org/10.1002/lary.25345 (2016).

26. Lee, J. et al. Effect of both local and systemically administered dexamethasone on long-term hearing and tissue response in a Guinea pig model of cochlear implantation. 18, 392-405 (2013).

27. Murphy, D., Daniel, S. J. J. O. H. \& Surgery, N. Intratympanic dexamethasone to prevent cisplatin ototoxicity: a guinea pig model. 145, 452-457 (2011).

28. Baulieu, E. E. et al. Steroid hormone receptors. Vitamins and hormones 33, 649-736 (1975).

29. Terunuma, T., Kawauchi, S., Kajihara, M., Takahashi, S. \& Hara, A. Effect of acoustic stress on glucocorticoid receptor mRNA in the cochlea of the guinea pig. Brain research. Molecular brain research 120, 65-72 (2003).

30. Rarey, K. E., Gerhardt, K. J., Curtis, L. M. \& ten Cate, W. J. Effect of stress on cochlear glucocorticoid protein: acoustic stress. Hear Res 82, 135-138 (1995).

31. Mori, T., Fujimura, K., Yoshida, M. \& Suzuki, H. Effects of glucocorticoid receptor antagonist on CAPs threshold shift due to shortterm sound exposure in guinea pigs. Auris, nasus, larynx 31, 395-399, https://doi.org/10.1016/j.anl.2004.09.008 (2004).

32. Hirose, Y. et al. The effects of the glucocorticoid receptor antagonist RU486 and phospholipase A2 inhibitor quinacrine on acoustic injury of the mouse cochlea. Neurosci Lett 413, 63-67, https://doi.org/10.1016/j.neulet.2006.11.029 (2007).

33. Tahera, Y. et al. NF-kappaB mediated glucocorticoid response in the inner ear after acoustic trauma. Journal of neuroscience research 83, 1066-1076, https://doi.org/10.1002/jnr.20795 (2006).

34. Gola, H. et al. Reduced peripheral expression of the glucocorticoid receptor $\alpha$ isoform in individuals with posttraumatic stress disorder: a cumulative effect of trauma burden. 9, e86333 (2014).

35. Mazurek, B. et al. Stress induces transient auditory hypersensitivity in rats. Hearing research 259, 55-63, https://doi.org/10.1016/j. heares.2009.10.006 (2010)

36. Lee, C.-M., Kim, B.-Y., Li, L. \& Morgan, E. T. Nitric oxide-dependent proteasomal degradation of cytochrome P450 2B proteins. Journal of Biological Chemistry 283, 889-898 (2008).

37. Bodwell, J. E. et al. Identification of phosphorylated sites in the mouse glucocorticoid receptor. Journal of Biological Chemistry 266, $7549-7555$ (1991). 
38. Heinrich, U. R. et al. Noise exposure alters cyclooxygenase 1 (COX-1) and 5-lipoxygenase (5-LO) expression in the guinea pig cochlea. Acta Otolaryngol 130, 358-365, https://doi.org/10.1080/00016480903168066 (2010).

39. Heinrich, U. R., Strieth, S., Schmidtmann, I., Stauber, R. \& Helling, K. Dexamethasone prevents hearing loss by restoring glucocorticoid receptor expression in the guinea pig cochlea. 126, E29-34, https://doi.org/10.1002/lary.25345 (2016).

40. Heinrich, U. R., Strieth, S., Schmidtmann, I., Stauber, R. \& Helling, K. Dexamethasone prevents hearing loss by restoring glucocorticoid receptor expression in the guinea pig cochlea. Laryngoscope, https://doi.org/10.1002/lary.25345 (2015).

41. Okret, S., Poellinger, L., Dong, Y. \& Gustafsson, J.-A. Down-regulation of glucocorticoid receptor mRNA by glucocorticoid hormones and recognition by the receptor of a specific binding sequence within a receptor cDNA clone. Proceedings of the National Academy of Sciences 83, 5899-5903 (1986).

42. Dong, Y., Poellinger, L., Gustafsson, J. A. \& Okret, S. Regulation of glucocorticoid receptor expression: evidence for transcriptional and posttranslational mechanisms. Mol Endocrinol 2, 1256-1264, https://doi.org/10.1210/mend-2-12-1256 (1988).

43. Wang, Y. et al. Changes of inflammatory cytokines and neurotrophins emphasized their roles in hypoxic-ischemic brain damage. The International journal of neuroscience 123, 191-195, https://doi.org/10.3109/00207454.2012.744755 (2013).

44. Hirose, K., Discolo, C. M., Keasler, J. R. \& Ransohoff, R. Mononuclear phagocytes migrate into the murine cochlea after acoustic trauma. The Journal of comparative neurology 489, 180-194, https://doi.org/10.1002/cne.20619 (2005).

45. Fujioka, M., Okano, H. \& Ogawa, K. Inflammatory and immune responses in the cochlea: potential therapeutic targets for sensorineural hearing loss. Frontiers in pharmacology 5, 287, https://doi.org/10.3389/fphar.2014.00287 (2014).

46. Nicolaides, N. C., Galata, Z., Kino, T., Chrousos, G. P. \& Charmandari, E. The human glucocorticoid receptor: molecular basis of biologic function. Steroids 75, 1-12, https://doi.org/10.1016/j.steroids.2009.09.002 (2010).

47. Samarasinghe, R. A., Witchell, S. F. \& DeFranco, D. B. Cooperativity and complementarity: synergies in non-classical and classical glucocorticoid signaling. Cell Cycle 11, 2819-2827, https://doi.org/10.4161/cc.21018 (2012).

48. Spahn, J. D. \& Szefler, S. J. In Pediatric Respiratory Medicine 219-233 (Elsevier, 2008).

49. Reichardt, H. M. et al. DNA binding of the glucocorticoid receptor is not essential for survival. Cell 93, 531-541 (1998).

50. Lowenberg, M., Stahn, C., Hommes, D. W. \& Buttgereit, F. Novel insights into mechanisms of glucocorticoid action and the development of new glucocorticoid receptor ligands. Steroids 73, 1025-1029, https://doi.org/10.1016/j.steroids.2007.12.002 (2008).

51. Weichhart, T. et al. The anti-inflammatory potency of dexamethasone is determined by the route of application in vivo. Immunology letters 129, 50-52 (2010).

52. Tuckermann, J. P. et al. Macrophages and neutrophils are the targets for immune suppression by glucocorticoids in contact allergy. The Journal of clinical investigation 117, 1381-1390 (2007).

53. Sadikot, R. T. et al. High-dose dexamethasone accentuates nuclear factor- $\kappa$ B activation in endotoxin-treated mice. American Journal of Respiratory and Critical Care Medicine 164, 873-878 (2001).

54. Han, W. K. et al. Susceptibility of Diabetic Mice to Noise Trauma. BioMed research international 2018 (2018).

\section{Acknowledgements}

Sponsored by funding from the Chungnam National University (2017-2204-01) to YHP.

\section{Author Contributions}

S.H.L. and A.R.L.: conceptualized/hypothesized, designed, performed the experiments; analyzed data; and prepared figures. S.A.S.: assisted with experiments and performed biochemical assays. S.H.J. and S.A.L.: assisted with experiments and animal handling. M.J.P.: refined data analysis and interpretation, prepared figures, and wrote manuscript. Y.H.P.: refined hypothesis and experimental design, data analysis and interpretation and wrote manuscript.

\section{Additional Information}

Competing Interests: The authors declare no competing interests.

Publisher's note: Springer Nature remains neutral with regard to jurisdictional claims in published maps and institutional affiliations.

Open Access This article is licensed under a Creative Commons Attribution 4.0 International License, which permits use, sharing, adaptation, distribution and reproduction in any medium or format, as long as you give appropriate credit to the original author(s) and the source, provide a link to the Creative Commons license, and indicate if changes were made. The images or other third party material in this article are included in the article's Creative Commons license, unless indicated otherwise in a credit line to the material. If material is not included in the article's Creative Commons license and your intended use is not permitted by statutory regulation or exceeds the permitted use, you will need to obtain permission directly from the copyright holder. To view a copy of this license, visit http://creativecommons.org/licenses/by/4.0/.

(C) The Author(s) 2019 